Digital Press Social Sciences and Humanities

La chanson folklorique enfantine comme media de l'apprentissage interculturel et du transfert des valeurs morales

Sri Handayani

Proceeding of Conférence internationale sur le français 2018

Joesana Tjahjani, Merry Andriani, Sajarwa, Wening Udasmoro (eds) 


\title{
La chanson folklorique enfantine comme media de l'apprentissage interculturel et du transfert des valeurs morales
}

\author{
Sri Handayani \\ Universitas Negeri Semarang, Semarang,Indonesia \\ e-mail : srihandayani@mail.unnes.ac.id
}

\section{Résumé}

La chanson folklorique enfantine est un exemple des produits culturels ayant plusieurs buts dans sa création. A part pour s'amuser, ce type de chanson a pour but aussi de transférer des valeurs morales aux enfants. Grace à ses valeurs morales, la chanson enfantine est un moyen potentiel comme media de l'apprentissage du caractère et aussi l'apprentissage interculturel. Cet article a pour but de proposer une compilation des chansons enfantines javanaises traduites en français destinée pas seulement aux apprenants indonésiens apprenants le français, mais aussi aux francophones qui sont en train d'apprendre la langue indonésienne. La recherche met l'accent sur le processus de traduire la parole de la langue javanaise en français. Ce processus est une assimilation de deux cultures différentes en s'accentuant sur la promotion de la culture d'origine à travers la langue. Le grand objectif au futur est pour rendre les chansons traditionnelles plus connues dans le monde entier. Après avoir appliqué l'approche descriptive, sept chansons enfantines javanaises ont été traduites en français, ce sont : Gundhul pacul, Padhang mbulan, Menthok-menthok, Dhondhong opo salak, Sluku-sluku bathok, Lir ilir, et Siji loro telu. Chaque chanson a sa valeur et contient aussi des éléments culturels. Le livret et le CD contenant la traduction des chansons enfantines javanaises en français sont les produits finals de la recherche qui pourrait être profité dans le transfert interculturel indonésien-français et en même temps l'éducation morale.

\section{Mots-clés}

Apprentissage interculturel, chanson folklorique, valeurs morales

\begin{abstract}
Children's folk song is an example of cultural products that has several goals in its creation. Aside from entertaining, this type of song also aims to transfer moral values or character building to children. Thanks to its moral values, this type of song is a potential medium as a media of character learning and also intercultural learning. This article aims to propose a compilation of Javanese children's folk songs translated into French intended not only for Indonesian learners who learn French, but also for French speakers who learn Indonesian language. The research focused on the process of translating the Javanese folk songs into French. This process is an assimilation of two different cultures by accentuating on the promotion of the culture of origin through the language. The future big goal is to make traditional songs better known around the world. After applying the developmental research approach, seven Javanese children's folk songs have been translated into French: Gundhul pacul, Padhang mbulan, Menthokmenthok, Dhondhong opo salak, Sluku-sluku bathok, Lir ilir, and Siji lor otelu. Each song has its value and also contains cultural elements. A booklet and a CD containing the translation of Javanese children's folk songs into French is the final product of this research that could be used in intercultural learning and at the same time character building
\end{abstract}

\section{Keywords}

Moral value, children's folk song, intercultural 


\section{Introduction}

La chanson est un moyen de divertissement amusant. Les chansons peuvent être utilisées comme supports d'apprentissage pour fournir du matériel d'apprentissage. A l'intérieur de la chanson il y a des éléments, tels que la notation, le rythme, l'harmonie et les paroles. La chanson est également l'un des produits culturels comme un exemple de l'art, l'un des éléments culturels. Ce produit culturel a un caractère universel. Parfois, il y a une chanson connue non seulement par les habitants dans un pays, mais aussi dans certains pays et chantée dans plusieurs langues. Par exemple, en Indonésie il y a la chanson « Burung hantu » dont les paroles sont :

\section{«Matahari terbenam, hari mulai malam, Terdengar burung hantu suaranya merdu Kukuk, kukuk, kukukkukukkukuk, Kukuk, kukuk, kukukkukukkukuk»}

Les Français connaissent aussi ce type de chanson comme une chanson enfantine dont le titre est « Dans la forêt lointaine ». La parole de cette chanson est comme suit :

« Dans la forêt lointaine, on entend le hibou, Du haut de son grand chêne, il répond au coucou Coucou hibou, coucou hibou, coucou hibou, coucou, Coucou hibou, coucou hibou, coucou hibou coucou»

En remarquant les deux paroles, il y a un concept similaire dans la chanson «Burung hantu » et « Dans la forêt lointaine ». Les deux chansons ont le même rythme, la même idée d'histoire, qui concerne le hibou. Les deux pays reconnaissent les hiboux comme des oiseaux qui sortent la nuit et vivent dans de grands arbres, qui ont la voix «kukuk (coucou)»

De l'exemple ci-dessus, nous pouvons voir qu'il existe des similitudes entre les concepts culturels de l'Indonésie et ceux de la France, bien que ce pays soit séparé par des milliers de kilomètres, et situés sur le différent continent en ayant des caractéristiques et des contextes culturels différents.

Cette étude vise à proposer une compilation de chansons folkloriques indonésiennes traduites en français. La chanson pourrait être utilisée comme un moyen d'introduction et d'apprentissage de la culture franco-indonésienne, premièrement pour les apprenants du français en Indonésie. Deuxième public cible de cette chanson est les Français vivant en Indonésie ou apprenant l'indonésien afin qu'ils puissent se rapprocher et savoir plus la culture indonésienne de moyen amusant. La chanson folklorique des enfants se compose de plusieurs lignes et parfois il y a un message à transmettre à travers la chanson. Par conséquent, la chanson est considérée appropriée pour introduire des connaissances interculturelles.

La chanson folklorique fait partie des chansons régionales qui a des caractéristiques simples utilisant des échelles pentatoniques, elle est traditionnelle car elle grandi de la culture locale, est transmis des parents à leurs enfants, chanté pendant que les enfants jouent, souvent anonyme et sans compositeur, et qui n'est pas destiné à des fins commerciales (Purnomo \& Subagyo 2010, p. 5).

Les chansons folkloriques javanaises qui sont traduites en français montrent une forme d'assimilation de la diversité, à savoir la culture indonésienne et française. Cette assimilation culturelle est l'un des efforts pour gérer l'hétérogénéité culturelle appliquée dans différents pays, tel que proposé par l'INRP (2007, p. 13) qu'il existe quatre modèles : le modèle d'assimilation, d'intégration, multiculturel et interculturel. Par conséquent, les politiques éducatives sont appliquées pour y répondre sous diverses formes, parmi lesquelles la préservation de la culture autochtone et l'éducation à la diversité. L'objectif principal de l'assimilation culturelle est de promouvoir les cultures indigènes dans l'apprentissage des langues étrangères. Dans ce cas, les chansons folkloriques javanaises en tant que la culture indigène de l'Indonésie, sont considérées comme le média de promotion, à travers l'outil de la langue française.

L'objectif à long terme à atteindre pour que la chanson folklorique enfantine puisse être appréciée par les apprenants du français en Indonésie et aussi par les locuteurs natifs du français en Indonésie et dans le monde entier. De plus, pour amener la chanson folklorique indonésienne à être connue dans le monde entier.

Certains résultats des études précédentes ont servis comme référence. Les symboles et significations de la nationalité dans les paroles de chansons folkloriques de Java Central et sa mise en œuvre dans le monde de l'éducation. Les résultats de cette étude indiquent que 1) le symbole et le sens des paroles de la 
chanson folklorique comme une manifestation de l'esprit du nationalisme ont une pertinence dans l'aspect éducatif de l'éducation, celle la nation et de la terre, du caractère et de la culture; 2) la mise en œuvre des symboles et la signification de la nationalité des chansons folkloriques dans l'éducation formelle peut être faite à travers l'enseignement de l'appréciation littéraire sur le cours de la langue javanaise.

« Le message culturel de la chanson folklorique à Java central » est également étudié par Arini Hidayah, publié dans Journal de l'Education volume $22 \mathrm{n}^{\circ}$ I Mars 2013. Selon Hidayah, les chansons folkloriques à Java Central comme Dhondhong opo salak, Gundhul pacul, et Kupu kuwi ont une signification culturelle soit dans l'éducation et soit dans la vie des enfants, entre autres sont les 3 caractères humains illustrés tels que le fruit Dhondhong, zalacca et doukou. Le meilleur des trois illustrations est le fruit de $d u k u$ en raison de ses bonnes qualités intérieures et extérieures, subtiles et douces.

Julia et Supriyadi ont mené une recherche sure « L'héritage des valeurs dans la chanson Sundanaise de Cianjur à Java Ouest » (2017, p. 120). Ils ont trouvé que la chanson sundanaise de Cianjur comprend des valeurs historiques, religieux, amoureux de la nature et de l'humanité.

Suharto et al ont examiné « Les chansons de Banyumas comme la réflexion de caractère des gens de Banyumas » (2017, p. 49). En général, les paroles des chansons traditionnelles de Banyumas sont drôles et rafraîchissantes en utilisant le dialecte banyumasan. Les paroles de la chanson montrent le caractère de la société et les gens de Banyumas et leur idéologie explicitant dans le langage utilisé, telles que l'ouverture d'attitude et de parole, l'honnêteté à la vérité qu'ils voient.

Les quatre études ont un rapport avec le sujet parlé dans cet article, à savoir : les chansons folkloriques contiennent des valeurs culturelles et peuvent être appliquées dans l'éducation. La différence se trouve dans la livraison de ces chansons en français et l'objectif n'est pas seulement d'éduquer les personnages, mais aussi d'apprendre les connaissances interculturelles.

L'apprentissage interculturel est un processus qui exige les apprenants de se connaître, de connaître leurs origines avant de comprendre les autres (Gillert, 2007, p. 32). L'objectif de l'apprentissage interculturel d'Oullet cité par Guedes (2007, p. 34) est de cultiver une compréhension de la culture de la société moderne, la capacité de communiquer avec des personnes de cultures différentes, une grande flexibilité dans le contexte de la diversité culturelle et un sentier culturel commun.

Selon Ouellet cité par Guedes (2007, p. 34), l'éducation interculturelle peut être conçue dans le but de promouvoir et de développer :1) une meilleure compréhension des cultures dans les sociétés modernes ; 2) une capacité de communication renforcée entre les membres de différentes cultures ; 3) une plus grande flexibilité dans le contexte de diversité culturelle qui caractérise la société ; 4) une plus grande capacité de participation à l'interaction sociale et la reconnaissance du patrimoine commun de l'humanité.

Dans l'apprentissage interculturel, les apprenants sont fournis de la capacité de répondre à de nouvelles cultures, d'exprimer leurs points de vue et de comparer cette nouvelle culture avec leur propre culture. Les buts finals sont donc : 1) enseigner aux apprenants les compétences linguistiques et socioculturelles; 2) préparer les apprenants à prendre en contact avec des gens ayant la culture différente; 3) apprendre aux apprenants à avoir un caractère ouvert et accepter des gens des cultures variés.

\section{Méthode}

Cette recherche est une recherche descriptive utilisant la méthode d'ethnographie. L'ethnographie est la première forme de compréhension d'autres phénomènes, impliquant diverses manières d'écrire en relation avec la collecte de données, telles que les coutumes et la langue (Ratna 2016, p. 86). Les étapes prises dans la recherche sont:

1. Choisir et déterminer des chansons folkloriques enfantines de Java Central. Comme elles sont très nombreuses, il faut choisir celles qui sont les plus connues des gens d'aujourd'hui. Finalement, il y a sept chansons sélectionnées, ce sont : Gundhul pacul, Padhang mbulan, Menthok-menthok, Dhondhong opo salak, Sluku-sluku bathok, Lir ilir, et Siji loro telu.

2. Analyser les valeurs morales dans les chansons folkloriques. Dans cette étape, la théorie à laquelle je fais référence est la théorie de l'éducation du caractère du Ministère de l'éducation.

3. Identifier le contenu culturel dans les chansons folkloriques en se basant sur la théorie des éléments culturels, enrichi d'autres sources telles que l'Internet. 
4. Traduire les paroles des chansons folkloriques de la langue javanaise en français en tenant compte de l'acceptabilité des paroles, non seulement en fonction de la structure de la langue.

\section{Résultat et discussion}

Après avoir appliqué l'approche descriptive, sept chansons enfantines javanaises ont été analysées ses valeurs morales et culturels et ensuite traduites en français, ce sont : Gundhul pacul, Padhang mbulan, Menthok-menthok, Dhondhong opo salak, Sluku-sluku bathok, Lir ilir, et Siji loro telu. Chaque chanson a son valeur et contient aussi des éléments culturels.

\subsection{Gundhul Pacul}

Parole originale :

Gundhul gundhul pacul, gembelengan

Nyunggi nyunggi wakul, gembelengan

Wakul ngglèmpang, segane dadi saklatar

Wakul ngglèmpang segane dadi saklatar

La parole de chanson folklorique Gundhul-gundhul pacul décrit un enfant chauve, méchant, têtu, arrogant et irresponsable. Il ne peut pas distinguer les bonnes et les mauvaises choses. Il pense qu'il est le plus juste, le plus capable et le plus intelligent, alors il est arrogant et ignorant. A cause de son arrogance, tout est finalement tombé et s'effondre.

L'élément culturel trouvé dans la chanson est:

- Pacul est l'instrument utilisé par le paysan pour cultiver le sol. Il représente le prolétaire dont la majorité est le paysan.

- Wakul est un panier en bambou pour mettre le riz. Ce panier est un produit typique de Java. Les javanais ont l'habitude de mettre le riz pour que le riz ait la saveur de la nature.

- Le riz est le repas principal de la majorité des Indonésiens.

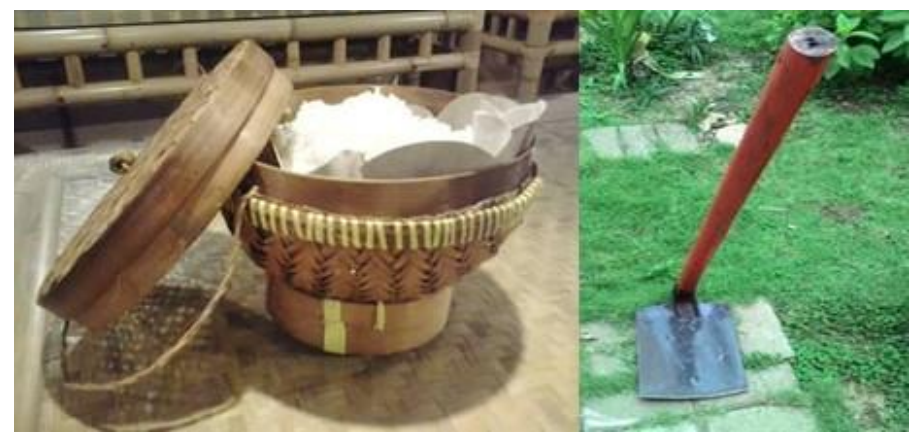

Figure 1 Wakul et Pacul

La version Française de la parole est comme suit:

Le garçon de tête chauve, il hoche sa tête

Le panier sur la tête, il hoche sa tête

Le panier tombe, les riz sont dispersés partout 


\subsection{Menthok-menthok}

Parole originale :

Menthok, menthok tak kandhani

Mung lakumu angisin isini

Mbokya aja ngetok ana kandhang wae

Enak enak ngorok oran yambut gawe

Menthok, menthok mung lakumu

Megal megol gaw eguyu

La parole de cette chanson dit que menthok ou canard est un symbole de la coquetterie et de la paresse. Sa façon de marcher en tortillant des hanches montre sa coquetterie. Il parait qu'il veut séduire les autres à travers cette attitude. Il préfère dormir que travailler signifiant qu'il est paresseux. Cependant, même s'il est paresseux, méchant et aime dormir, mais qu'il a au moins un bon côté, qu'il peut faire rire les autres.

'Menthok-menthok' voudrait transférer le message sur le regard en soi. En tant qu'être humain, il est évident d'éviter d'être arrogant et paresseux. La paresse cause de mauvaises influences. Il vaut mieux d'essayer de travailler dur pour fournir les besoins de la vie.

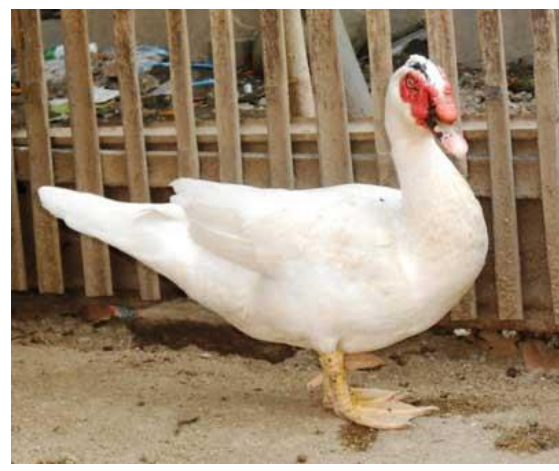

Figure 2 Menthok

La parole en français est comme suit :

Canard, coquette, écoute-moi,

Tu marches comme ça, à gauche et à droite,

Ne sois pas dans ta cage, feignante et paresseuse,

Tu dors toute la journée, ne fais pas de travail

Canard, coquette, et quand tu marches,

A gauche à droite, ça nous fait rire

\subsection{Padhang mbulan}

Yo prakanca dolanan ing jaba,

Padhang mbulan padhange kaya rina

Rembulane e sing awe-awe,

Ngelingake aja padha turu sore

Ya prakanca dhapadha mrene,

Bareng-bareng dolanan suka-suka

Langite padhang sumebar lintang,

Ya padha dolanan sinambi cangkriman 
La valeur religieuse existe dans la parole de Padhang mbulan. Les gens sont invités de rappeler et remercier toujours le Dieu en profitant la nature. Il vaut mieux de ne pas dormir tôt pour pouvoir faire la prière le soir. Jadis, le village n'a pas été équipé de l'électricité. Quand il y avait la pleine lune, les gens sortaient de leurs maisons pour regarder la lune, se réunir avec des voisins, joués avec des amis, sous la lumière de la lune. Le sens de l'amitié et de l'unité se reflète dans la parole.

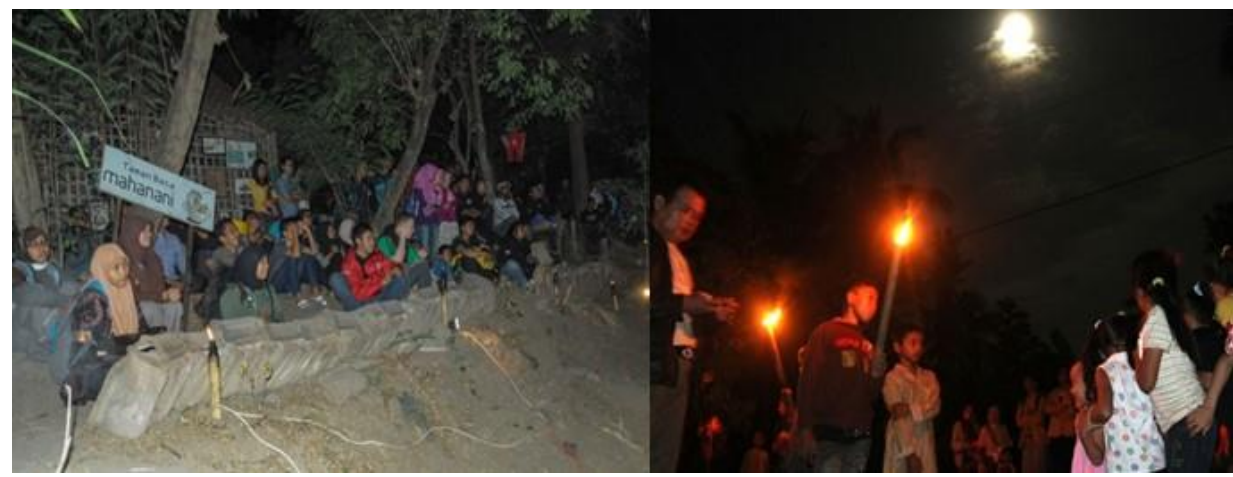

Figure 3 Activités sous la pleine lune

Voici donc la parole en français :

Mes chers amis, allons jouer au jardin

La lune est belle, la nuit est comme le jour

Regardez la lune, nous invite toujours

Elle nous rappelle de ne pas nous coucher très tôt

Mes chers amis, venez vite vous approcher

Allons jouer ensemble avec la joie

Le ciel est clair et il est plein d'étoiles

Nous l'apprécions en faisant la devinette

\subsection{Dhondong opo salak}

Dhondhong opo salak, duku cilik-cilik

Ngandhong opo mbecak, mlaku timik-timik

Adhik ndherek ibu, tindak menyang pasar

Ora pareng nakal ora pareng rewel

Mengko ibu mesthi mundhut oleh-oleh

Gedhang karo roti, adhik diparingi

La chanson parle des caractères des gens représentés des trois fruits typiques de l'Indonésie : Dhondhong, salak et duku. Dhondhong est un fruit tropique appelé aussi le pomme Cythère, ayant le goût acide. Ce fruit est lisse à la partie extérieure mais rugueuse et aiguisé dans la partie intérieure. La philosophie représentée par ce fruit est qu'il existe des gens qui ont de bon air, mais ils ont de mauvais but et mauvais cœur. Salak ou zallacca est appelé aussi le fruit de serpent en raison de sa peu rugueuse et pointue. Mais à l'intérieure, ce fruit a la couleur claire et la texture croustillante. Cette description représente des gens dont leur qualité sont couverts par leur apparence féroce. Tandis que doukou a de bonne apparence soit à l'extérieur soit à l'intérieur. Selon la comparaison des caractères de trois fruits, le meilleur est si les gens se comportent comme le fruit de doukou qui dit et fait comme ce qu'ils montent à l'extérieur.

Alors que la parole dans la troisième ligne parle de moyen de transport traditionnel : Andhong et becak. Andhong est un type de chariot tiré par un cheval. Tandis que becak est un moyen de transport de trois roues profitant de la force humaine pour le faire marcher. La phrase a pour but de transférer la valeur de l'indépendance, de marcher sur nos propres pieds, de ne pas déranger et troubler les autres pour réaliser notre affaire. Pendant le processus de réalisation, il faut être patient et ne pas être pressé. Si nous le faisons, nous obtiendrons de bons résultats. 


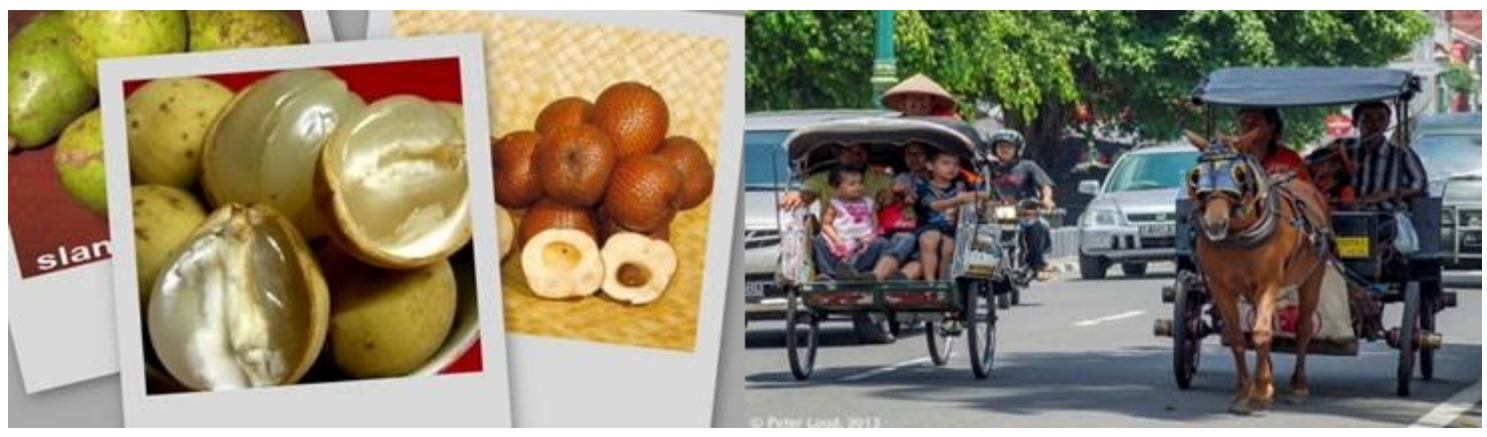

Figure 4 Dhondong, Salak, Andhong et Becak

Voici la traduction de la chanson en français :

Un Dhondhong ou un zalaque, ou de petiits doukous

Aller en chariot, en pédicap, ou marcher en petit pas

Tu vas avec maman, pour aller au marché

Mais ne sois pas méchant, ne sois pas impatient

Maman achètera, beaucoup de fruits des mains

Des bananes et des gâteaux, je te donnerai un peu

\section{Conclusion}

La chanson est un œuvre universel ayant plusieurs fonctions, y compris le média de transfert de messages moraux et de messages culturels. La traduction des chansons folkloriques javanaises en français a pour but d'inviter les apprenants du français en Indonésie à apprécier mieux la culture traditionnelle, d'attirer les locuteurs natifs du français ou les francophones pour apprendre la culture indonésienne, et d'amener la chanson folklorique indonésienne à être connue dans le monde entier.

\section{Références}

Gillert, A. (2007). Concept de l'apprentissage interculturel. In T-kit L'appprentissage interculturel. Brussels: Conseil de l'Europe.

Guedes, M. de J. C. (2007). A propos d'éducation interculturelle. In T Kit l'apprentissage interculturel. Brussels: Conseil de l'Europe.

Hidayah, A. (2013). Makna Budaya Lagu Dolanan di Jawa Tengah. Jurnal Pendidikan, 22(1).

Institut National de Recherche Pédagogique. (2007). Approche interculturelle en éducation: étude comparative internationale. Paris: INRP.

Julia, \& Supriyadi, T. (2017). The inheritance of values in Sundanese song of Cianjuran in West Java.

Harmonia: Journal of Arts Research and Education, 17(2), 120-128.

https://doi.org/10.15294/harmonia.v17i2.8645

Purnomo, W., \& Subagyo, F. (2010). Terampil Bermusik. Jakarta: Pusat Perbukuan Nasional.

Ratna, N. K. (2016). Metodologi Penelitian Kajian Budaya dan Ilmu Sosial Humaniora pada Umumnya.

Yogyakarta: Pustaka Pelajar. 
Suharto, S., Sumaryanto, T., Ganap, V., \& Santosa, S. (2016). Banyumasan Songs as Banyumas People's Character Reflection. Harmonia: Journal of Arts Research and Education, 16(1), 49-56.

https://doi.org/10.15294/harmonia.v16i1.6460 